\title{
La conformación de un campo editorial global: el nuevo escenario para la circulación de las ideas y su impacto en el mercado argentino
}

\author{
The conformation of a global publishing field: the new scenario for \\ the circulation of ideas and its impact in the Argentinian market
}

ARTÍCULO

\author{
Daniela Páez \\ Universidad Nacional de Quilmes, Argentina. \\ Contacto: cd.paez@outlook.com
}

Recibido: septiembre de 2017

Aceptado: octubre de 2017

\begin{abstract}
Resumen
El presente artículo se enfoca en analizar las condiciones de circulación de libros e ideas del campo editorial global, un espacio que está integrado por múltiples actores con desiguales capacidades de acción e influencia en el sistema, que comenzó a consolidarse a partir de la década de 1980. Asimismo, se hace hincapié en cómo esta dimensión transnacional repercutió directamente en el mercado argentino, en un sector altamente concentrado compuesto por un puñado de grupos editoriales multinacionacionales. En este sentido, se hace necesario caracterizar a ese fenómeno como un rasgo propio de un escenario que excede los límites nacionales y que está signado por la constitución -y constante reconfiguración- del espacio global.

Palabras claves: campo editorial; libros; industrias culturales; concentración económica.

Abstract

This article will set focus on analyzing the conditions of book circulation, and therefore of ideas, in the global publishing field, a space composed by multiple actors with unequal capacities for action and influence within the system, that began to consolidate from the 1980s. It will also emphasize how this transnational dimension had a direct impact on the Argentine market, a sector highly concentrated in a handful of multinational publishing groups. In this sense, it is necessary to characterize this phenomenon as a characteristic of a scenario that exceeds national boundaries and which is marked by the constitution - and constant reconfiguration - of the global space.
\end{abstract}

Keywords: publishing field; books; cultural industries; economic concentration. 


\section{El nuevo campo editorial global}

Este espacio editorial, que trasciende las fronteras y las culturas nacionales, comenzó a conformarse a partir de la década de 1980, en el marco del proceso de globalización de la economía, que promovía el libre comercio de bienes y servicios, y de la irrupción de las tecnologías digitales, que modificaron los hábitos de consumo y las lógicas de producción de las industrias culturales en su conjunto. En este escenario emergieron como actores de peso los multimedios internacionales. Estos funcionan próximos al polo comercial y controlan grandes cuotas de los mayores mercados del libro; en el extremo más cultural se encuentran las pequeñas y medianas firmas que operan a nivel local o encaran políticas de exportación a baja escala y no compiten directamente con los principales actores, sino que suelen aprovechar nichos de mercado o espacios que estos consideran poco rentables. Sin embargo, no es posible hablar de relaciones de poder equilibradas, ya que los grandes grupos imponen criterios productivos frente a los cuales el resto debe desarrollar estrategias específicas para mantenerse competitivos y autosustentables, tanto en los canales alternativos como en el circuito comercial. Tampoco se trata de dos categorías opuestas y fácilmente caracterizables: en conjunto conforman un campo que no resulta uniforme ni indiferenciado sino que los actores se ubican entre ambos puntos en función de condiciones particulares, propias y de sus entornos. En este sentido, algunos autores prefieren hablar de una mundialización del campo editorial, para oponer la diversidad y heterogeneidad de este espacio frente a la homogeneidad y estandarización que propone el concepto de globalización (Escobar, 2005; Szpilbarg, 2012). Retomando a Sapiro (2009), José de Souza Muniz Jr. (2016) y Daniela Szpilbarg (2012), destacan que esta etapa de mundialización está signada por las asimetrías evidenciadas en la disputa por el control de los mercados, entre los países centrales y entre estos con aquellos de la periferia. Dujovne \& García (2011) agregan que "la idea de literatura mundial no puede pensarse sino a condición de comprender las desigualdades históricas entre centros idiomáticos y culturales de mayor poder, y una heterogénea periferia conformada por zonas más débiles" (p. 31). Por lo tanto, la posición de los actores en el espacio editorial queda definida por cuestiones que trascienden las fronteras nacionales y están más vinculadas a la pertenencia a los mercados dominantes, que se erigieron en esa posición gracias a una trayectoria cultural, política y social.

De esta manera, dentro del sistema tienden a consolidarse determinadas características específicas que influyen en la relación entre sus integrantes y las lógicas productivas que 
desarrollan: una creciente concentración de los mercados en manos de un grupo reducido de actores hegemónicos y la emergencia de otros menos poderosos, que no orientan su producción al público masivo ni esperan obtener enormes niveles de rentabilidad; la internacionalización de la cadena de valor del libro en función del abaratamiento de costos; la aparición de otro tipo de capitales, no siempre vinculados a las industrias culturales, que comienzan a invertir en el rubro de la producción de libros y fomentan la aplicación de las nuevas técnicas económicas y financieras a los modelos tradicionales de negocio; la revalorización de figuras especializadas como los traductores, directores de colecciones y agentes literarios; y el posicionamiento de los espacios internacionales de intercambio como puntos clave donde confluyen los diferentes flujos de circulación de libros, de ideas y personas. Por su parte, insertas en esta estructura global, las dinámicas de producción, circulación y consumo en los mercados locales están igualmente determinadas por características propias de ese ambiente: condiciones económicas y demográficas del país; la historia, tradición y grado de profesionalización del sector; las particularidades del público, su poder adquisitivo y la tasa de alfabetización; y el rol adoptado por el Estado para promover la lectura, difundir internacionalmente su cultura, fomentar el desarrollo de su industria editorial e impulsar las exportaciones y traducciones. Estos factores también inciden de diferente manera en la posición y el peso de los actores nacionales en el espacio mundial (Dujovne, 2016; De Souza Muniz Jr., 2016).

En este marco, como reacción a la liberalización de la producción y comercialización de libros, a principios de los ochenta surgió el concepto de bibliodiversidad, que remite a la necesidad de resistir a las lógicas de funcionamiento de los mercados globalizados y a incentivar políticas que promuevan la diversidad cultural en el mundo de la edición (Saferstein \& Szpilbarg, 2014). Bajo el patrocinio de la Unesco, desde hace tres décadas la Alianza de Editores Independientes es uno de los principales promotores de esta bandera, que en nuestro país es enarbolada por Editores Independientes de la Argentina por la Diversidad Bibliográfica (EDINAR). Estos colectivos reconocen la importancia del actor político para preservar la pluralidad de ideas y tienen por objetivos fomentar las políticas públicas acerca del libro, la digitalización de la lectura, la lectura juvenil, la publicación de autores locales y han sido importantes portavoces en la defensa de la edición independiente (Alianza Internacional de Editores Independientes, 2014).

\section{La concentración del mercado editorial}


Uno de los primeros en advertir problema de la concentración en el sector fue André Schiffrin en su libro de La edición sin editores publicado en 1999 (2001), donde propone que durante la última década del siglo XX la estructura del campo editorial sufrió una trasformación sin precedentes: tradicionalmente, el mundo de la edición había funcionado sobre la base de un modelo de negocio que valorizaba fuertemente el capital simbólico como medio de rentabilidad, y poco se había mezclado con otros sectores económicos y financieros. Sin embargo, el escenario cambió radicalmente con la consolidación y expansión de los grandes conglomerados multimediales que, diversificados en varios sectores productivos, rápidamente comenzaron a controlar los circuitos internacionales de circulación de bienes culturales.

La inversión de otro tipo de capitales ajenos el sector editorial generó verdaderas trasformaciones en los modelos productivos, ya que se fijó como objetivo el aumento de las tasas de rentabilidad para equipararlas a las ganancias generadas por sus otros sectores de inversión, como la prensa gráfica o el área audiovisual. Al absorber a una firma mediana o pequeña, describe Schiffrin, el multimedio anunciaba su intención de mantener la autonomía del sello y sus tradiciones editoriales, sin embargo, prontamente aparecían trasformaciones comerciales, económicas y literarias que apuntaban a homogeneizar a todos los miembros del grupo. Así, los criterios de edición comenzaron a acercarse al polo más comercial y se popularizó la publicación de best-sellers, de libros escritos por figuras del star system televisivo, o cualquier estrategia que prometiera vender grandes tiradas a un público masivo y seguro (2001).

El tipo de estructura económica de las empresas multinacionales, si bien presenta un alto grado de heterogeneidad, posee un rasgo característico: su tendencia a la concentración en pos de ampliar su influencia en los mercados globalizados. Alberto Pérez Gómez (2000) cita a A. Van Loon y A. Lange (1991) para definir ese fenómeno de la siguiente manera:

Podemos hablar de concentración de medios de comunicación cuando se produce: a) una fusión de empresas de comunicación, esto es, la absorción de una empresa por otra o la asociación de dos o varias para formar una sola; o b) una integración empresarial, es decir, toda forma de adquisición de propiedad o de poder que afecte a las actividades de una sociedad o grupo de sociedades (p. 81).

En términos más generales, el autor agrega que, independientemente de los movimientos de capital de los actores, la concentración también puede asociarse a una preponderancia en el mercado con respecto a los otros competidores:

Existe concentración en un mercado cuando una empresa alcanza una determinada 
posición de influencia en el mismo, con independencia de si ello se debe a una fusión, a la adquisición de otras empresas o a la creación de joint ventures de cualquier tipo (crecimiento externo), o de si se debe al resultado positivo de su propia actividad o al incremento, por cualquier motivo, de su cuota de mercado (crecimiento interno) (p. 81).

Asimismo, Pérez Gómez distingue entre diferentes tipos de concentración bajo los cuales puede funcionar el mercado de bienes culturales y clasifica a estas empresas de acuerdo a su organización. En primera instancia, define como "integración horizontal (monomedia)" a un grupo o empresa que controla en un mercado varias unidades de producción en la misma fase del proceso productivo y los productos ofrecidos resultan idénticos o sustitutivos; otro caso es el de la "integración vertical", donde un actor controla varias etapas del proceso productivo; la "integración multimedia" se observa cuando una empresa posee varios tipos de medios de comunicación; en la "integración multisectorial (conglomerados)" un grupo controla simultáneamente medios de comunicación y otras actividades económicas; finalmente, se habla de "integración internacional" cuando una empresa de comunicación opera a la vez varios mercados nacionales (2000, p. 82). También resulta pertinente para este trabajo la propuesta de Kojima (1973) sobre la clasificación de las empresas multinacionales basada en las estrategias comerciales que adoptan a la hora de realizar inversiones en países extranjeros: las multinacionales "orientadas al comercio" (tradeoriented) buscan aprovechar las ventajas comparativas que ofrecen los mercados de sus filiales extranjeras, especialmente en aquellos sectores que comienzan a debilitarse en el país de origen; y las "sustituidoras de importaciones" (anti-trade-oriented) orientan su producción al mercado en el que establecen sus filiales y aprovechan las ventajas oligopólicas que les permiten dominar los mercados internacionales por medio de la preponderancia generalizada en los mercados locales (pp. 2-4).

\section{Los grandes actores del campo editorial global}

En la actualidad el mundo de la edición es controlado mayoritariamente por cinco grandes compañías que forman parte de conglomerados multimediales: Hachette Book Group, Holtzbrinck/Macmillan, Penguin Random House, Harper Collins y Simon \& Schuster; durante las décadas del 80 y el 90, este escenario se encontraba en pleno proceso consolidación. Para comienzos del siglo XXI los actores de mayor peso en el campo eran fondos originarios de Europa y Estados Unidos. En Alemania se afianzaba el poderoso Grupo Bertelsmann, que hasta 1988 (cuando tuvo lugar la fusión entre Time Inc. y Warner Communications) había sido el mayor multimedios del mundo. En Francia, Group de la Cité y Hachette concentraban el 
$50 \%$ de la edición en ese país aunque sólo el último había desarrollado una estrategia de inversión internacional como parte de Lagardère Group. Pearson y Reed Group se posicionaban en Gran Bretaña y en los Estados Unidos, además de Random House, que desde 1998 formaba parte del grupo alemán; se afianzaban aquellas firmas que habían recibido inversiones de las grandes corporaciones audiovisuales, como la tradicional editorial neoyorquina Simon and Schuster, que se había sumado al imperio Paramount Communications a finales de los 80 (Sabouret, 2002).

\section{El mercado del libro en lengua castellana}

A nivel local, la transformación de la estructura del campo editorial estuvo vinculada principalmente a la extranjerización y concentración del mercado español y a la expansión del Grupo Bertelsmann. Se trata de dos factores interrelacionados, ya que sellos en vías de expansión como Planeta, Santillana y Ediciones B debieron volcarse hacia los mercados latinoamericanos en parte para no perder competitividad frente al gigante alemán (Vanoli, 2009), que a partir de la compra Plaza \& Janés había comenzado a ganar terreno en el mercado del libro en lengua castellana. Sin embargo, resulta destacable que los conglomerados de origen español no lograron posicionarse como líderes en el campo de la edición global. Una explicación puede encontrarse en el poco peso relativo de ese mercado frente a otros como Estados Unidos, China, Alemania, Japón, Francia y Reino Unido, cuyo consumo conjunto de libros asciende al $60 \%$ de la producción mundial (International Publishers Association [IPA], 2013). Esto también evidencia la disputa que mantienen los centros idiomáticos sobre la base de desigualdades históricas y culturales que posicionan a unos como dominantes y relegan a otros a la periferia (Dujovne \& García, 2011). ${ }^{1}$

\footnotetext{
${ }^{1}$ Dujovne (2016) ilustró esta situación a partir de un trabajo etnográfico realizado en la Feria Internacional de Frankfurt: divididos por temas y regiones idiomáticas, los stands más visitados fueron en primer lugar el inglés, seguido del alemán y el francés y, bastante más lejos, el italiano y el español. Después seguían las lenguas asiáticas, que, aunque se editan en mercados gigantescos, mantienen una circulación local. Los menos visitados eran aquellos que promocionaban la edición árabe, persa y africana.
} 
Divulgatio. Perfiles académicos de posgrado, Vol. 2, Número 4, 2017, 104-119.

\begin{tabular}{|c|c|}
\hline Ferias del Libro más visitadas por profesionales de la & edición \\
\hline 2014 & 2015 \\
Frankfurt & Frankfurt \\
London & London \\
$\begin{array}{c}\text { Bologna/BookExpo } \\
\text { America }\end{array}$ & Bologna \\
Guadalajara & Guadalajara \\
Beijing/Sharjah & Paris \\
Paris & BookExpo America \\
Abu Dhabi & Beijing \\
Delhi & Gothenburg (Göteborg) \\
Istanbul & Moscow/Seoul/Taipei \\
Cairo & Delhi/lstanbul \\
\hline
\end{tabular}

Elaboración propia en base a IPA (2016)

Si bien la fase de concentración comenzó a acelerarse a partir de la década de 1980, el comienzo temprano de la internacionalización de las editoriales españolas también contribuyó a fortalecer su presencia en los países hispanohablantes del continente gracias al conocimiento que acumularon durante décadas sobre esos mercados locales. En general, antes de convertirse en actores dominantes, estas firmas participaban en ellos mediante dos estrategias: la exportación-importación, es decir, un acuerdo comercial de representación con determinadas distribuidoras, como fueron los casos de Anagrama y el Grupo Océano; y la apertura de filiales comerciales y productivas en los mercados nacionales de interés -como Brasil, México y Argentina a la cabeza, seguidos de Colombia, Chile y Venezuela-, para poder producir a precios locales e introducir temas y autores nacionales en los catálogos a fin de generar una mejor respuesta a la demanda local. (Fernández Moya, 2009). Ya en proceso de expansión, los multimedios encontraron escenarios nacionales muy favorables para el desembarco de sus inversiones gracias al triunfo de regímenes democráticos que aplicaron políticas neoliberales centradas, de acuerdo a las recomendaciones del Consenso de Washington, en las privatizaciones de los servicios públicos, la flexibilización de la economía, una fuerte retracción de la intervención estatal y el endeudamiento con el Fondo Monetario Internacional.

\section{Los principales actores internacionales en el mercado argentino}

Durante la década de 1990, el sector de las industrias culturales locales se encontraban atravesando su propio proceso de transformación. Tal como describen Mastrini y Becerra (2009), las diferentes crisis económicas desalentaron la compra de sus productos - de manera 
selectiva- por parte de las familias que buscaban reducir gastos. Mientras tanto, la expansión de la industria de las telecomunicaciones facilitó la popularización de la circulación digital de contenidos, a través de las descargas online y vía streaming; así, tuvo lugar una resignación del soporte y los hábitos de consumo comenzaron a inclinarse hacia las tecnologías digitales: "si bien en algunas industrias (como la prensa escrita) se constatan retrocesos vinculados, entre otras causas, como el avance de nuevos soportes (como Internet) otros sectores, como la telefonía móvil, de modo notable, se caracterizan por su expansión” (p. 207). En este contexto de baja competitividad internacional y crisis económica, entre la década de 1990 y los primeros años del siglo XXI las editoriales nacionales más competitivas habían pasado a formar parte de firmas extranjeras: Tesis en 1991, Kapelusz en 1994, Sudamericana en 1998, Emecé y Minotauro en 2001 y Paidós en 2003. Así, la mayor parte del mercado argentino comenzó a ser controlado por cuatro grandes conglomerados multinacionales: los grupos Planeta (Seix-Barral, Ariel, Espasa-Calpe y Editorial Emecé); Bertelsmann (Plaza \& Janés, Lumen, Grijalbo y Sudamericana); y, con menor preponderancia, el español PRISA-Santillana (Alfaguara, Aguilar y Taurus) y el colombiano Norma (Tesis y Kapelusz).

El rol del Estado como desregulador de la economía jugó un papel clave. Durante esta época no hubo políticas públicas directas de fomento al libro y la lectura, por el contrario, se recortaron beneficios impositivos que hasta ese momento se aplicaban al sector. También repercutieron ostensiblemente las políticas económicas que caracterizaron al gobierno de Carlos Saúl Menem, y que afectaron al conjunto de las industrias culturales: la apertura de mercado, el incentivo a la inversión extranjera, la privatización de los servicios públicos y el Plan de Convertibilidad y la devaluación del peso de fines de la década disminuyeron aún más la competitividad de la industria nacional, que ya venía intentado superar las crisis económicas de las décadas del setenta y ochenta y sufría un importante atraso de su planta industrial. De esta forma, el paso al costado del actor estatal se concreta dando lugar a políticas de autorregulación, fuertemente marcadas por los intereses del actor empresarial y basadas en la lógica mercantil.

En general, los grandes grupos que comenzaron a marcar lógicas de producción y circulación de libros importaron modelos y estrategias de negocios de las casas matrices que apuntaban a aumentar la rentabilidad en un corto espacio de tiempo y aplicaron lógicas productivas basadas en criterios más comerciales. De esta manera, al finalizar la década del 90, Argentina se había integrado a la cadena global de valor del libro mediante la venta de las principales firmas locales y se había concretado una importante reconfiguración del mercado nacional y de sus lógicas de funcionamiento. También comenzaron a surgir otros actores, 
cuyo fin no era competir en el canal comercial con estos nuevos gigantes, sino que aprovechaban los nichos de mercado e innovaban en modelos de gestión que les permitiesen circular también en canales alternativos: el sector de los sellos conocidos como independientes. A continuación se ahondará en la historia y las características de esas primeras multinacionales que tomaron el control del mercado nacional, y que mantienen su posición -con excepción de Norma S.A.- hasta nuestros días.

\section{Grupo Planeta}

Planeta, el grupo español que en el año 2000 se había consolidado como el principal actor del mercado argentino, fue fundada en Barcelona en 1949 como una editorial dedicada principalmente a la literatura. Su fundador, José Manuel Lara Hernández, fue un editor que supo mantener una buena relación con el régimen de Franco y prosperó gracias a su visión ambiciosa en los negocios. En 1952 creó el Premio Planeta que, con una primera dotación económica de 40 mil pesetas, resultó un verdadero éxito para nutrir el catálogo con varias plumas de renombre, generar capital simbólico y promocionar las obras de ganadores y finalistas. La casa comenzó una rápida expansión que incluyó la apertura de filiales en Hispanoamérica y la inversión en otras áreas productivas. De acuerdo a Sergio Vila-Sanjuán (2003), para mediados de la década de 1960 ya había diversificado sus actividades en el rubro editorial sumando textos educativos, volúmenes de consulta y grandes obras, y a partir de los setenta comenzó a editar fascículos gracias a una joint venture con la firma italiana De Agostini. Durante los últimos años de esa década inició un proceso de innovación mediante la aplicación técnicas de marketing, estudios de mercado, el aumento de la inversión en publicidad y la definición de los precios no en función del costo del libro, sino del público al que iba dirigido. Su expansión en Europa comenzó en el año 1982 con la compra del 70\% de la firma que había sido su principal competidora durante los años del franquismo: Seix Barral, que se había fusionado con editorial Ariel en los 70 y se encontraba en quiebra al momento de la venta. Con esa adquisición, los Lara advirtieron la rentabilidad de comprar editoriales como medio para engrosar el catálogo y acrecentar las ventas. La estrategia que aplicaron fue ofrecer servicios centralizados pero que cada sello funcione de manera autónoma y tenga sus propios equipos editoriales y de marketing. En 1986 sumó Ediciones Deusto y el $50 \%$ de otra de sus mayores rivales en el mercado español, Editorial Destino. En 1991 compró el 50\% de Espasa-Calpe, lo que le permitió obtener el control de la cadena de librerías Casa del Libro. 
En 1994 compró el 40\% de Tusquets aunque sólo lo mantuvo hasta $1998^{2}$ y la asociación definitiva entre ambas empresas llegó recién en 2012. En Argentina, además de su filial local, Planeta desembarcó mediante una alianza con editorial Sudamericana a principios de los ochenta (que fue disuelta a los pocos años) pero el movimiento definitivo lo dio en el año 2000 cuando compró Editorial Emecé; al año siguiente también adquirió editorial Minotauro (de Diego, 2012).

\section{Grupo Bertelsmann}

El nacimiento de este multimedio se remonta a 1835, a la pequeña localidad de Gütersloh en Alemania, una pequeña editorial que comenzó publicando biblias y se consolidó durante el nazismo. Su expansión fuera del mercado local se originó a partir de la idea del gerente Reinhard Mohn de crear el Círculo de Lectores, una suerte de club de lectura que hoy cuenta con 29 millones de miembros en todo el mundo (Usi, 30 de septiembre de 2005). El grupo inició un temprano proceso de expansión, diversificación y transnacionalización que traspasó los límites del mundo editorial. En 1958 fundó las compañías Ariola Records y Sonopress, y en 1968 ingresó al mundo periodístico con una participación del $25 \%$ en la cadena Grüne \& Jahr, la firma de impresión y edición más grande de Europa, porcentaje que en 1976 ascendió al 74.9\%. En 1979 le compró Arista Records a Columbia y en 1986 adquirió la mayoría de RCA Records, movimiento mediante el cual creó Bertelsmann Music Group, para concentrar sus inversiones en el mundo de la música. ${ }^{3}$ Hasta finales de los 80 , Bertelsmann fue el multimedio más poderoso del mundo pero descendió al segundo lugar después de la fusión entre Time-Warner en 1988; no obstante, continuó liderando el mercado editorial y mantuvo el grueso de su facturación originada en publicación de libros, mientras que los otros grandes del podio triunfaban en el terreno de la producción audiovisual (Sabouret, 2002). El posicionamiento del grupo en el mercado del libro en lengua castellana comenzó en 1977 con la compra del 40\% de Plaza \& Janés (inicialmente había desembarcado en España en 1962 con su Círculo de Lectores). En 1982, gracias a la derogación por parte

\footnotetext{
${ }^{2}$ La editorial, fundada por Beatriz de Moura y Óscar Tusquets en 1969, recuperó el porcentaje vendido a Planeta en abril de 1998, aunque en noviembre de ese año vendió el $50 \%$ de sus acciones a la firma RBA, que había nacido en 1981 por obra del editor argentino Ricardo Rodrigo, la agente literaria Carmen Balcells y el ex director de Editorial Bruguera, Roberto Altarriba. A principios de 2000 el sello recuperó esa participación y quedó bajo la dirección de Moura y Antonio López Lamadrid (El País, 16 de marzo de 2000). Finalmente, en 2012 volvió a asociarse con Planeta.

${ }^{3}$ BGM Entertainment se unió con Sony Music Entertainment y formó la poderosa joint venture Sony BMG, que funcionó entre 2003 y 2008.
} 
del gobierno español de la ley que impedía que capitales extranjeros posean la totalidad de una empresa nacional, Bertelsmann compró el $60 \%$ restante. A través de esa firma, el grupo centralizó el manejo de sus editoriales en ese mercado y realizó otras compras estratégicas como el 80\% del paquete accionario de Lumen en 1996 (Vila-Sanjuán, 2003; de Diego, 2012). En nuestro país la consolidación de Bertelsmann llegó de la mano de la adquisición del 60\% de Editorial Sudamericana en 1998.

Mediante la compra de Random House en 1998, la editorial más importante del mercado estadounidense, Bertelsmann logró hacerse con el dominio definitivo sobre el mercado editorial internacional. Hasta ese momento, RH pertenecía a Advance Publications, propiedad del magnate de las comunicaciones Newhouse Jr. De acuerdo a André Schiffrin (2001), para el año 1997, Random superaba los 80 millones de dólares en pérdidas, aunque, antes de decidir la venta, Newhouse había intentado aumentar las ganancias mediante una serie de cambios en la orientación comercial del sello y estrategias como el pago de adelantos extraordinarios a figuras mediáticas que pudieran atraer al público masivo. El resultado fue el opuesto y las ventas rara vez cubrían el costo inicial: "Newhouse había logrado la hazaña de arruinar el capital intelectual de la casa, disminuir su reputación y perder dinero al mismo tiempo" (p. 92); en este escenario, Bertelsmann oficializó la compra por unos mil millones de dólares. En 2001 Random House realizó un acuerdo de edición y comercialización con la editorial italiana Mondadori, que desde 1991 pertenecía al grupo Finivest, parte del multimedios de Silvio Berlusconi, y era dueña del sello español Grijalbo desde 1985 y del italiano Einaudi desde 1994. En 2013 Bertelsmann continuó su expansión mediante una de las fusiones más importantes en el ámbito de la edición durante de los últimos años, entre su sello Random House y Penguin Group, propiedad de Pearson PLC.

\section{Grupo PRISA-Santillana}

PRISA-Santillana fue otro de los conglomerados internacionales que se posicionó como uno de los líderes en el mercado local a fines de los noventa. Editorial Santillana, la firma reconocida a nivel internacional por editar libros escolares y material educativo, fue fundada en 1960 por Jesús de Polanco y Francisco "Pancho" Pérez González, quienes más tarde inauguraron el sello de literatura infantil y juvenil Altea. Tres años después abrieron una filial en nuestro país, en 1968 otra en Nueva York y continuaron por ese camino hasta llegar a tener presencia en 22 países. El proceso de expansión del grupo continuó en 1974 con la compra de editorial Taurus, que había nacido en 1954 de la mano del propio Pérez González 
junto a Rafael Gutiérrez Girardot y Miguel Sánchez López. Siguió con las compras de la madrileña Alfaguara en 1980 y de Editorial Aguilar en 1986 (Vila-Sanjuán, 2003; de Diego, 2012). En el año 2000, dos años después de crear el Premio Alfaguara de Novela, el sello se incorporó al Grupo PRISA, ${ }^{4}$ multimedios que edita los periódicos The Huffington Post, El País y Le Monde, entre otros, y tiene inversiones en radio, televisión, telefonía y publicidad. En 2016 compró Carvajal Soluciones Educativas por 16,8 millones de euros, que incluía a empresas como Kapelusz, Greenwich, Buenas Noches, Torre de Papel, Zona Libre, Educa Inventia, Voluntad y la licencia para utilizar la marca Norma (El País, 2 de marzo de 2016)

\section{Grupo Carvajal}

La editorial colombiana Norma fue fundada en 1960 por el Grupo Carvajal, que había comenzado sus actividades en 1904 con una imprenta comercial en Cali y, con el transcurso del tiempo, fue ampliando su producción hasta lograr posicionarse internacionalmente como proveedores de artículos y muebles de oficina, papelería, impresión, entre otros. En el campo de la edición literaria el sello tuvo un espectacular éxito en su país gracias a la adquisición de los derechos para publicar las obras de Gabriel García Márquez; a partir de ese momento, se propuso replicarlo en el resto del mercado latinoamericano y posicionarse como referente en el mundo de la edición. Antes de invertir en Argentina había comenzado a operar en Ecuador desde 1987; en 1990 había adquirido la editorial española Parramón y, durante el mismo año, había iniciado la distribución de su producción en Chile. El ingreso en el mercado local lo realizó en 1991 mediante la adquisición de un porcentaje de Editorial Tesis y la apertura de una filial a través de la cual en 1994 compró Kapelusz, el tradicional sello de libros y manuales escolares (Carvajal International S.A., 2009). Sin embargo, su preponderancia duró poco más de una década, ya que la gerencia general de Carvajal no logró compatibilizar su proyecto comercial con una buena adaptación al mundo del libro y al funcionamiento de los mercados latinoamericanos. Finalmente, las pérdidas constantes incentivaron el cierre de las líneas de literatura y ensayo en 2001, y la eventual venta de Carvajal Soluciones Educativas al Grupo PRISA en 2016. Editorial Norma, que nunca había sido una inversión, quedó relegada y el

\footnotetext{
${ }^{4}$ En Argentina el Grupo PRISA sumó la compra de Radio Continental en 2005 y se convirtió en accionista del canal Telefé mediante la adquisición en 2006 de la empresa española Telefónica Internacional. Ese mismo año comenzó a emitir localmente la cadena española de radiodifusión Los 40. También es socio de Papel Prensa junto con el Grupo Clarín y La Nación.
} 
grupo colombiano continuó centrado en otros sectores más redituables, como la producción de papelería, celulosa o los artículos para oficina.

\section{Conclusiones}

La conformación del espacio editorial global implicó el desarrollo de nuevas lógicas productivas y de consumo que influyeron, e influyen, en la circulación de libros e ideas. Este espacio se encuentra en constante reconfiguración, ya que los actores no permanecen estáticos, sino que se mueven en función de las posibilidades de aumentar su influencia en los mercados; en este contexto, el crecimiento mediante compras y fusiones se vuelve una condición necesaria para alcanzar márgenes de rentabilidad que nunca fueron propios del sector. De esta manera, los grandes grupos implementan estrategias de internacionalización más agresivas mientras que los sellos independientes se mantienen locales o encaran políticas de exportación a baja escala. Sin embargo, el campo mundial del libro no se estructura en dos frentes diferenciados, sino que los actores que lo conforman coexisten y se relacionan en el mismo espacio, aunque con proyectos editoriales y comerciales diferentes. Asimismo, aparece una amplia variedad de modelos de negocio y nuevos canales de circulación de libros, alternativos pero no excluyentes al tradicional-comercial.

El concepto de bibliodiversidad irrumpe en este escenario en oposición a la estandarización de los consumos culturales y a la bestsellerización. Así, el rol del Estado se vuelve más relevante para fomentar la lectura, impulsar la industria editorial local y defender la pluralidad de ideas al no dejar la producción cultural librada a los avatares del mercado. Sin embargo, para analizar la constitución de este espacio global no resulta pertinente reducir las relaciones que se establecen en su interior en términos de oposición entre el polo cultural, que busca la diversidad, y el comercial que promueve la bestsellerización, sino que la verdadera oposición que lo estructura y que realmente condiciona los flujos de circulación se da entre los centros idiomáticos y mercados dominantes (con el inglés en primer lugar) y los espacios periféricos dependientes.

\section{Referencias bibliográficas}

Alianza Internacional de Editores Independientes (2014). Declaración Internacional de los Editores Independientes para contribuir a la defensa y promoción de la bibliodiversidad. Recuperado de: 
http://www.allianceediteurs.org/IMG/pdf/declaration_internacional_de_los_editores_independie ntes_2014.pdf

Becerra, M. \& Mastrini, G. (2009). Los Dueños de la Palabra. Buenos Aires: Prometeo Libros.

Carvajal International S.A. (2009). Informe Anual 2009. Recuperado de: http://www.carvajal.com/wp-content/uploads/2011/01/Informe-Anual-20091.pdf

Kojima, K. (1973). A Macroeconomic Approach to Foreign Direct Investment. Hitotsubashi Journal of Economics 14, 1-21. Recuperado de: http://econpapers.repec.org/article/hithitjec/v_3a14_3ay_3a1973_3ai_3a1_3ap_3a1-21.htm

De Diego, J. L. (2012). Concentración Económica, Nuevos Editores, Nuevos Agentes. Coloquio Argentino de Estudios sobre el Libro y la Edición. Universidad Nacional de La Plata: FaHCE, Argentina. Recuperado de: http://coloquiolibroyedicion.fahce.unlp.edu.ar/actas/de_Diego/view

De Souza Muniz Jr., J. (2016). Debates sobre globalización editorial. Cursos Virtuales Instituto de Desarrollo Económico y Social, Buenos Aires.

Dujovne, A. (2016). Frankfurt, hablame de mí. Anfibia. Recuperado de: http://www.revistaanfibia.com/ensayo/frankfurt-hablame-de-mi/

Dujovne, A. \& García, D. 2011. Introducción a la "Literatura mundial". Políticas de la Memoria, $(10,11,12), 31-37$.

Escobar, A. (2005). Más allá del tercer mundo. Globalización y diferencia. Bogotá: Instituto Colombiano de Antropología e Historia.

Fernández Moya, M. (2009). Editoriales españolas en América Latina. Un proceso de internacionalización secular. Información Comercial Española (849), pp. 65-77. Recuperado de: $\quad$ http://www.revistasice.com/CachePDF/ICE_849_6578_8847B920642349ACFD0111B6E4AF2C9D 
International Publishers Association (2016). IPA World book fair report 2016. Recuperado de: https://www.internationalpublishers.org/images/datastatistics/IPAWorldBookFairReport2016.pdf

International Publishers Association (2013). IPA Global Publishing Statistics. Recuperado de: http://www.internationalpublishers.org/images/data-statistics/2012/ipa-global-publishingstatistics-2.pdf

Pérez Gómez, A. (2000). Las concentraciones de medios de comunicación. Quaderns del Consell de I'Audiovisual de Catalunya 7, 81-91.

Sabouret, Y. (2002). The place of multimedia groups in the world of publishing. En Fred Kobrak \& Beth Luey (ed.). The structure of international publishing en the 1990s. New Jersey: Transaction Publishers.

Sapiro, S. (2011). El espacio intelectual en Europa entre los siglos XIX y XXI. Políticas de la memoria, $(10,11,12), 57-65$.

Saferstein, E. \& Szpilbarg, D. (2014). La industria editorial argentina, 1990-2010: entre la concentración económica y la bibliodiversidad. Alter/Nativas Latin American Cultural Studies Journal, (3). Recuperado de: http://alternativas.osu.edu/es/issues/autumn2014/essays2/saferstein-szpilbarg.html

Schiffrin, A. (2001). La edición sin editores. Las grandes corporaciones y la cultura (Eduardo Gonzalo, trad.). México: Ediciones Era. (1ra ed. 1999)

Szpilbarg, D. (2012). ¿Es posible hablar de un 'campo editorial global'?: un análisis acerca de los agentes, mediaciones y prácticas en el espacio editorial trasnacionalizado. Coloquio Argentino de Estudios sobre el Libro y la Edición. Universidad Nacional de La Plata: FaHCE, Argentina. Recuperado de: http://coloquiolibroyedicion.fahce.unlp.edu.ar/actas/Szpilbarg.pdf/view

Vanoli, H. (2009). Pequeñas editoriales y transformaciones en la cultura literaria argentina. Apuntes de investigación del CECYP, (15), 161-185. Recuperado de: http://www.apuntescecyp.com.ar/index.php/apuntes/article/view/277 
Vila-Sanjuán, S. (2003). Pasando página. Autores y editores en la España democrática. Barcelona: Ediciones Destino.

\section{Artículos periodísticos}

Diario El País (2016). Grupo PRISA compra empresas del Grupo Carvajal. Recuperado de: http://www.elpais.com.co/economia/grupo-prisa-compra-empresas-del-grupo-carvajal.html

Diario El País (2000). Tusquets recupera la participación del 50\% que vendió a RBA. Recuperado de: http://elpais.com/diario/2000/03/16/cultura/953161221_850215.html

Deutsche Welle (2005). Bertelsmann: de las biblias al imperio mediático. Recuperado de: http://www.dw.de/bertelsmann-de-las-biblias-al-imperio-medi\%C3\%A1tico/a-1725524 